\title{
Numerical modelling of anaerobic digestion reactor dynamics with a delay
}

\author{
A. A. Khan ${ }^{1}$ J. J. Shepherd ${ }^{2} \quad$ A. J. Stacey ${ }^{3}$
}

(Received 14 January 2016; revised 13 July 2016)

\begin{abstract}
Anaerobic digestion of municipal and animal wastes provides a potentially important means of producing energy sustainably. The reliable production of methane from anaerobic digestion requires that bacteria metabolise municipal and agricultural wastes to produce mainly carbon dioxide and methane. Our model uses Monod based kinetics and is complex since the biomass concentration growth is inhibited with the increase in substrate. The production of methane depends on the number of methanogenic bacteria; there is evidence from previous chemostats experiments that there is a time delay in population number of these cells to respond to changes in substrate concentrations. In this article we focus on determining the dynamical response of the methane production rate under different dynamics of feed conditions
\end{abstract}

http://journal.austms.org.au/ojs/index.php/ANZIAMJ/article/view/10474 gives this article, (c) Austral. Mathematical Soc. 2016. Published August 15, 2016, as part of the Proceedings of the 12th Biennial Engineering Mathematics and Applications Conference. ISSN 1446-8735. (Print two pages per sheet of paper.) Copies of this article must not be made otherwise available on the internet; instead link directly to this URL for this article. 
and temperatures. Since there is a delay in the methane bacteria mass response, we include the effects of the delay on the dynamics of the system based on modification of kinetics in a simple fermentation anaerobic model. We compare the dynamics of the system with and without delay to show the contribution of delay to the overall stability of the system.

\section{Contents}

1 Introduction

C143

2 Governing equations

C145

2.1 The non-delay system . . . . . . . . . . . . . . . C146

2.1.1 Dimensionless formulation . . . . . . . . . . C147

2.1.2 Critical points . . . . . . . . . . . C C148

2.1.3 Numerical determination of critical points . . . . . C149

2.2 System incorporating a time delay . . . . . . . . . . C C150

2.2.1 Linear stability of the system with a time delay . . C151

3 Discussion and results

3.1 Scenario one . . . . . . . . . . . . . . C152

3.2 Scenario two . . . . . . . . . . . . . . C155

References

C157

\section{Introduction}

Anaerobic digestion of sewerage sludge, manure and municipal solid wastes results in the production of digester gas (biogas) that contains methane with the balance being principally carbon dioxide. Since this fuel resource is increasing with population, it is important to optimize the volumetric 
production rate of methane in order to maximize the power generation. This optimization is facilitated by utilizing mathematical models of the anaerobic digestion process. These models predict dynamics affecting the rate of methane generation. The dynamics analysis is also important since the start-up of anaerobic digesters is difficult [1], and dynamic models facilitate the choice of parameter identification.

The possibility of including delay terms into the differential equations in models of biological processes has received more attention recently, due to the increasing availability of analytical and numerical mathematical tools. Physically, such delays or lags represent gestation times or incubation periods. Complex biological processes are combined, accounting only for the time required for these processes to occur. Thingstad and Langeland [2] used this approach in a Monod model for a specific growth rate. They found that a time delay led to the prediction of autonomous oscillations, which were also observed experimentally. However, according Beddington and May [3], time delays by themselves are not necessarily destabilizing since other complex factors such as changes in the environment may affect the growth rates. More recently, San and Stephanopoulos [4] justified the inclusion of delays in their modelling of fermentation reactors, based on the observation of delays in the physical experiments by Stephanopoulos and San [5]. Their modelling indicated that a time delay has a significant effect on the dynamics, under certain conditions.

In a previous study [6] we optimized the methane production process for a biological system without delays. In the present study we focus on the change in the dynamics of a system caused by a time delay. We consider a simple model, developed by Andrews [7], in which the organic substrates are disintegrated and hydrolysed by a Monod type of kinetics. The reaction kinetics are based on two autonomous nonlinear differential equations that govern the concentrations of substrates $S$, and the concentration of bacteria $X$. In this, we assume that the methane generation is directly in proportion to the concentration of the bacteria. We modify this system by the introduction of a time delay in the appropriate kinetic term and determine its effect on 
the system's dynamics. We are particularly interested in the reaction startup (where problems for later stages of the reaction may arise), whether oscillations arise and how the behaviour of the system relates to that without delays. To our knowledge, this analysis of such a system with a time delay has not been done before.

We first consider the undelayed system, and show that, for suitable data, the system has a pair of stable constant limiting states, with solutions tending monotonically to these states as time progresses. We show that, for the same data and a range of selected delays, solutions of the delayed system tend to these same limiting states, but exhibit oscillatory behaviour in the process.

\section{Governing equations}

The equations for the dynamic reactor modelling are based on those given by Andrews [10]. We choose a simple kinetics model involving two state variables, $S$ and $X$, to enable us to easily see the effects of time delays on the system dynamics. More sophisticated models exist, such as the ADM1 model proposed by Batstone et al [8]; however, these models have over 34 parameters that are difficult to fit for a model of a waste treatment facility. Therefore, we opt to use a simpler approach.

The state variables, $S$ and $X$, are functions of time $T$ and consequently are dependent variables. Five parameters control the process-substrate concentration of the feed $S_{F}$, biomass concentration of the feed $X_{F}$, hydraulic residence time $\theta$ (varied by changing the feed flow rate or the reactor volume), the initial substrate concentration $S_{0}$, and the initial biomass concentration $X_{0}$.

We consider two distinct situations. First, the non-delay case arises when all varying quantities depend on the current time T. Second, the delay case involves a delay of $\mathrm{T}^{*}$ in one of the varying terms. We subsequently show how these two cases are related. 


\subsection{The non-delay system}

The dimensional differential equations governing $S$ and $X$ are

$$
\begin{aligned}
& \frac{\mathrm{d} S}{\mathrm{dT}}=\frac{S_{\mathrm{F}}-\mathrm{S}}{\theta}-\frac{\mathrm{F}(\mathrm{S}) \mu \mathrm{X}}{\mathrm{Y}}, \\
& \frac{\mathrm{dX}}{\mathrm{dT}}=\frac{X_{\mathrm{F}}-\mathrm{X}}{\theta}-\mathrm{K}_{\mathrm{d}} \mathrm{X}+\mathrm{F}(\mathrm{S}) \mu \mathrm{X},
\end{aligned}
$$

where

$$
F(S)=\frac{1}{1+K / S+S / K_{i}} .
$$

The inhibition function $F(S)$ slows the rate of growth of the biomass, $\mu$ is the maximum specific growth rate, $K$ is the saturation coefficient, $K_{i}$ is the inhibition coefficient, $K_{d}$ is the micro-organism death rate $\left(\mu, K, K_{i}\right.$ and $K_{d}$ are the four kinetic constants), $\theta$ is the hydraulic residence time, $Y$ is the stoichiometric constant between the substrate and the biomass, and $S_{\mathrm{F}}$ and $\mathrm{X}_{\mathrm{F}}$ are the feed concentrations of the substrate and biomass, respectively.

The substrate concentration $S$ and biomass concentration $X$ in equations (1) and (2) refer to the biologically active food for the bacteria and the biomass concentration, respectively. There may be additional organics in the system that are considered inert.

The rate of change in substrate concentration $S$ is given in equation (1). The first term on the right-hand side is the change in substrate concentration caused by dilution from the feed concentration $S_{\mathrm{F}}$. The second term is the reduction of $S$ by the bacteria with the biomass concentration, $X$, in a chemical reaction with $\mu$ as the kinetic rate constant. In this model the kinetics is inhibited by an inhibition function $F$ which is a function of $S$ as given in equation (3).

The rate of change in biomass concentration is given in equation (2). On the right-hand side, the first term describes the reduction of bacteria concentration by dilution from the feed concentration $X_{F}$. The second on the right-hand 
side describes the reduction in biomass concentration caused by degradation. The third describes the increase in biomass caused by the population increase in $X$ with a reaction rate $\mu$ tempered by the inhibition function $F(S)$.

\subsubsection{Dimensionless formulation}

We transform the problem (1), (2) and (3) into dimensionless form by the transformations

$$
\begin{array}{ll}
t=\mu T, & s=\frac{S}{K}, \quad x=\frac{X}{Y K}, \quad s_{F}=\frac{S_{F}}{K}, \quad x_{F}=\frac{X_{F}}{Y K}, \\
a=\frac{K}{K_{i}}, \quad b=\frac{1}{\theta \mu}, \quad c=\frac{K_{d}}{\mu}, \quad s_{0}=\frac{S_{0}}{K}, & x_{0}=\frac{X_{0}}{Y K} .
\end{array}
$$

The resulting problem for the dimensionless state variables $s$ and $x$ is then

$$
\begin{aligned}
\frac{d s}{d t} & =b\left(s_{F}-s\right)-f(s) x, \\
\frac{d x}{d t} & =b\left(x_{F}-x\right)-c x+f(s) x, \\
s(0) & =s_{0}, \quad x(0)=x_{0},
\end{aligned}
$$

where

$$
f(s)=\frac{1}{1+1 / s+a s} .
$$

where $a, b$ and $c$ are the system parameters.

The system (5) and (6) is an autonomous highly nonlinear $2 \times 2$ system and the nonlinear initial value problem (5)-(7) is solved numerically using the Runge-Kutta algorithm in the ODE solver rk45 in Matlab [9]. 


\subsubsection{Critical points}

To determine the critical points (steady state solutions) $s_{\mathrm{cr}}$ and $x_{\mathrm{cr}}$ of the system (5) and (6) we need to solve the system

$$
\begin{array}{r}
b\left(s_{F}-s\right)-f(s) x=0, \\
b\left(x_{F}-x\right)-c x+f(s) x=0,
\end{array}
$$

where $f(s)$ is given in (8).

We find that the two solutions for the critical points are solutions to two cubic equations. The solutions are cast in terms of the parameters $a, b, c$, $s_{\mathrm{F}}$ and $x_{\mathrm{F}}$. To determine $s_{\mathrm{cr}}$ we need to solve the cubic equation for $s$,

$$
A_{1} s^{3}+A_{2} s^{2}+A_{3} s+A_{4}=0
$$

where

$$
\begin{aligned}
& A_{1}=a b+a c \\
& A_{2}=-a b s_{F}-a c s_{F}+b+c-1, \\
& A_{3}=-b s_{F}-c s_{F}+b+c+s_{F}+\chi_{F}, \\
& A_{4}=-b s_{F}-c s_{F} .
\end{aligned}
$$

Incorporating $\boldsymbol{f}(\boldsymbol{s})$ from (8) into (9), (10) and after some algebra, these equations reduce to a cubic equation in $s$. Solving this gives values for $s_{c r}$. Adding (9) and (10) gives

$$
x_{c r}=\frac{s_{\mathrm{F}}+x_{\mathrm{F}}-s_{\mathrm{cr}}}{(1+\mathrm{c} / \mathrm{b})}
$$

Values of all critical points depend on the five parameters $a, b, c, s_{F}$ and $x_{F}$. 
Table 1: The critical points of the non-delay system.

\begin{tabular}{c|rr} 
Pairs & $\mathrm{s}_{\mathrm{cr}}$ & $\chi_{\mathrm{cr}}$ \\
\hline (a) & 0.76 & 7.13 \\
(b) & 10.06 & -0.02 \\
(c) & 870.96 & -662.25
\end{tabular}

\subsubsection{Numerical determination of critical points}

With five operating parameters, we have much latitude to explore many possibilities, so we need a criterion to direct attention to a few cases of interest. Since we are interested in anaerobic digestion, in this study we base our parameter values on the experiments reported by Andrews [10]. Andrews [10] derives the parameter values $a=0.0015, b=0.3333$, and $c=0.1$. We also use the following values of the feed concentrations: $s_{\mathrm{F}}=10$ and $x_{F}=0.0333$. The value of $x_{F}$ is also derived from values used by Andrews. However, for the $s_{\mathrm{F}}$ value the main consideration is that it is much larger than $\chi_{F}$ so that shortage of food is not a constraint. We can check that the maximum value of $s$ does exceed $s_{\mathrm{F}}$.

Using these numerical values, we obtain the values for the three critical points of (5) and (6) as given in Table 1.

The stability of these critical points is determined from the solutions $\lambda$ of the quadratic equation

$$
\operatorname{det}\left[a_{i j}\left(s_{c r}, x_{c r}\right)-\lambda \delta_{i j}\right]=0, \quad i, j=1,2,
$$

where $a_{i j}\left(s_{c r}, x_{c r}\right)$ are the entries of the Jacobian matrix $J\left(s_{c r}, x_{c r}\right)$ of the system (5) and (6) evaluated at the critical points $\left(s_{\mathrm{cr}}, x_{\mathrm{cr}}\right)$ and $\delta_{i j}$ is the Kronecker delta. These solutions (eigenvalues) are displayed in Table 2.

Table 2 implies that both the critical points (a) and (c) are asymptotically stable, while (b) is unstable. Also (c) corresponds to a negative limiting $x$ value. While the critical point (c) does not represent a biologically real limiting 
Table 2: Numerical eigenvalues of Jacobian J.

\begin{tabular}{c|rr} 
Pairs & Eigenvalues of $J\left(s_{\mathrm{cr}}, \mathrm{x}_{\mathrm{cr}}\right)$ \\
\hline (a) & -2.14 & -0.46 \\
(b) & -0.33 & 0.37 \\
(c) & $-0.37+0.06 i$ & $-0.37-0.06 i$
\end{tabular}

state, its stability might imply that trajectories starting in the first quadrant (with $s_{0}, x_{0}>0$ ) cross the $s$ axis to reach (c), corresponding to the extinction of $x$. However, from (5) and (6), we see that at all points along the s-axis in the $(s, x)$ plane,

$$
\frac{d x}{d t}=b x_{F}>0 .
$$

Thus, any trajectory starting in the first quadrant cannot cross the s-axis, so (c) is an unreachable state for such initial values. Thus, $(a)$ is the only feasible limiting state for solutions starting with physically real initial values.

\subsection{System incorporating a time delay}

For reasons given in Section 1, there is evidence that for certain reactions involving bacterial cells, there is a time response which affects the dynamics of the system. Following the approach taken by Bush and Cook [11], we introduce a time delay in the inhibitory function $F(S)$ in the bacterial kinetics multiplication term of (2). We apply the transformations (4) to the resulting system, and arrive at the dimensionless system with incorporated delay

$$
\begin{aligned}
\frac{d s(t)}{d t} & =b\left(s_{F}-s(t)\right)-f(s(t)) x(t), \\
\frac{d x(t)}{d t} & =b\left(x_{F}-x(t)\right)-c x(t)+f(s(t-\tau)) x(t), \\
s(t) & =s_{0}, \quad x(t)=x_{0}, \quad \text { for } \quad t \in(-\tau, 0),
\end{aligned}
$$

where $\boldsymbol{f}(\mathrm{s})$ is given in (8), and $\tau=\mu T^{\star}$ is the dimensionless delay. For such a time delay problem we must not only include the initial conditions $s_{0}$ and $x_{0}$, 
but also provide a range of historical values of $s(t)$ and $x(t)$ for all the $t$ values in the interval $(-\tau, 0)$. Here we set $s(t)=s_{0}$ and $x(t)=x_{0}$ for $t \in(-\tau, 0)$.

Equations (19) and (20) form a nonlinear system of delay differential equations (DDEs) containing terms which draw on the values of solutions at previous times. The equations' nonlinearity means they must be solved numerically. However, the presence of the delay term in (20) means that the numerical technique used for the non-delay problem (5)-(7) is not appropriate, and an alternative method of numerically solving this system of DDEs must be used. Such an algorithm was only recently developed and is discussed in detail by Shampine et al. [12]. This method was included in the Matlab program dde23 and was tested successfully for a number of DDEs with constant time delays [12].

\subsubsection{Linear stability of the system with a time delay}

To investigate the stability of the delay system equations (19) and (20) need to be linearized about the critical points, $\left(\mathrm{s}_{\mathrm{cr}}, \mathrm{x}_{\mathrm{cr}}\right)$.

Here, stability of $\left(s_{c r}, x_{c r}\right)$ is determined from the solutions $\lambda$ of the transcendental equation

$$
\operatorname{det}\left[a_{i j}\left(s_{c r}, x_{c r}\right) e^{-\lambda \tau \delta_{2 j}}-\lambda \delta_{i j}\right]=0, \quad i, j=1,2,
$$

where $\boldsymbol{a}_{\mathrm{ij}}\left(s_{\mathrm{cr}}, \boldsymbol{x}_{\mathrm{cr}}\right)$ are as in (17) and $\tau$ is the time delay. Equation (22) reduces to (17) when delay is removed, that is, $\tau=0$. Equation (22) reduces to

$$
\mathrm{P}_{\tau}(\lambda) \equiv \lambda^{2}+\mathrm{P}\left(\mathrm{s}_{\mathrm{cr}}, \mathrm{x}_{\mathrm{cr}}\right) \lambda+\mathrm{Q}\left(\mathrm{s}_{\mathrm{cr}}, \mathrm{x}_{\mathrm{cr}}\right)+\mathrm{R}\left(\mathrm{s}_{\mathrm{cr}}, \mathrm{x}_{\mathrm{cr}}\right) \mathrm{e}^{-\tau \lambda}=0
$$

where $P, Q$ and $R$ are derived from $a_{i j}$. Solutions of (23) (when they exist) vary with $\tau$, and so will the stability of $\left(s_{\mathrm{cr}}, \mathrm{x}_{\mathrm{cr}}\right)$ as a limiting state of the delayed system (19), (20). Table 3 gives solutions for $\lambda$, corresponding to the critical point (a) of Table 1, for three values of delay $\tau$ and the data of Section 2.1.3. This shows that the critical point (a) of Table 1 is a stable limiting state of the delayed system for these delay values. 
Table 3: Solutions $\lambda$ of equation (23).

\begin{tabular}{r|r}
$\tau$ & $\lambda$ values \\
\hline 2 & $-1.236-3.162 i$ \\
3 & $-0.650-2.246 i$ \\
4 & $-0.407-1.740 i$
\end{tabular}

\section{Discussion and results}

In the previous sections we described two models representing anaerobic digester reactors. The first, described by equations (5)-(7), is autonomous, nonlinear and is solvable by standard numerical methods. In this model, information is drawn from values at the current time. The second model, given by Equations (19)-(21), is also nonlinear but is not autonomous; and information is drawn from present time values and also values from past times. Solutions of this model require a more sophisticated approach using recently developed methods. We apply the respective solution techniques to each of these models, using the data presented in Section 2.1.3, to obtain $x$, the bacteria or biomass and $s$, the substrate as functions of time. This is done for two scenarios, encountered in typical fermentation reactors and described below.

\subsection{Scenario one}

Here, we assume the reactor initially has no substrate or biomass present and has constant feeds of both substrates and biomass. We see this case as the main focus of our study since normally the feed stream contains both substrate and the biomass, such as in the case of sewage flow into a tank. It approximates a typical waste treatment system full of water which is displaced by entering feeds of biomass and substrates. Since the feed rate is constant and the liquid volume is constant, as it is controlled by overflowing, the 
Figure 1: Variation of biomass concentration for different time delays.

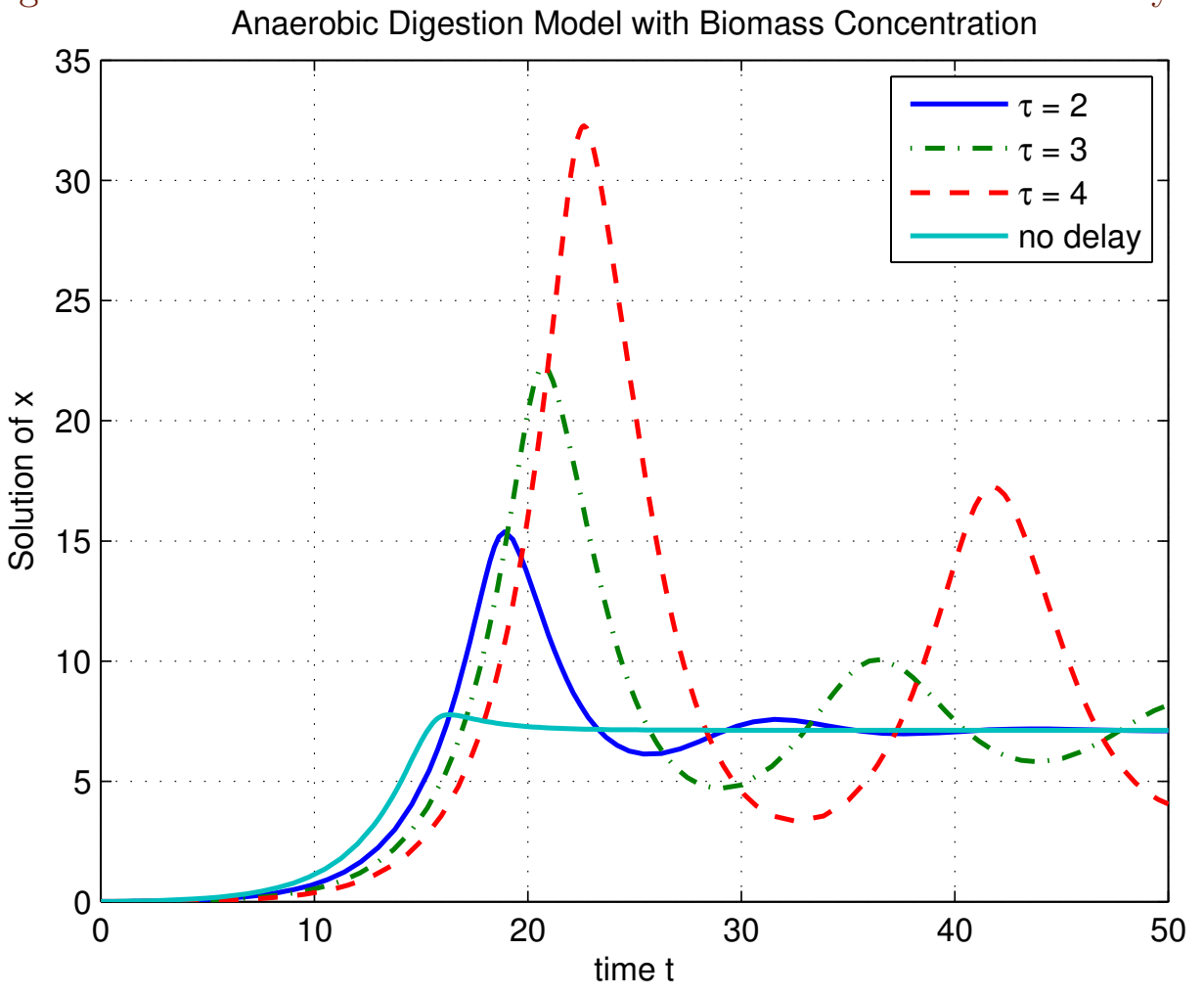

residence time $\theta$ of the slurry is constant. However, there is a transient response, since the concentrations of $s$ and $x$ change with time $t$.

Figures 1 and 2 show the time variation of $x$ and $s$ for Scenario one, as generated by the numerical solution methods described above. They show the results of no time delay (solved numerically by the Matlab solver rk45) and results for three different time delays $\tau$.

Figure 1 shows results for $x$ the biomass concentration. For the no delay case we see a smooth rise to a plateau value as $t$ is increased. This corresponds to the steady state value of $x=7.13$ as shown in Table 1 . Figure 2 shows 
Figure 2: Variation of substrate concentration for different time delays.

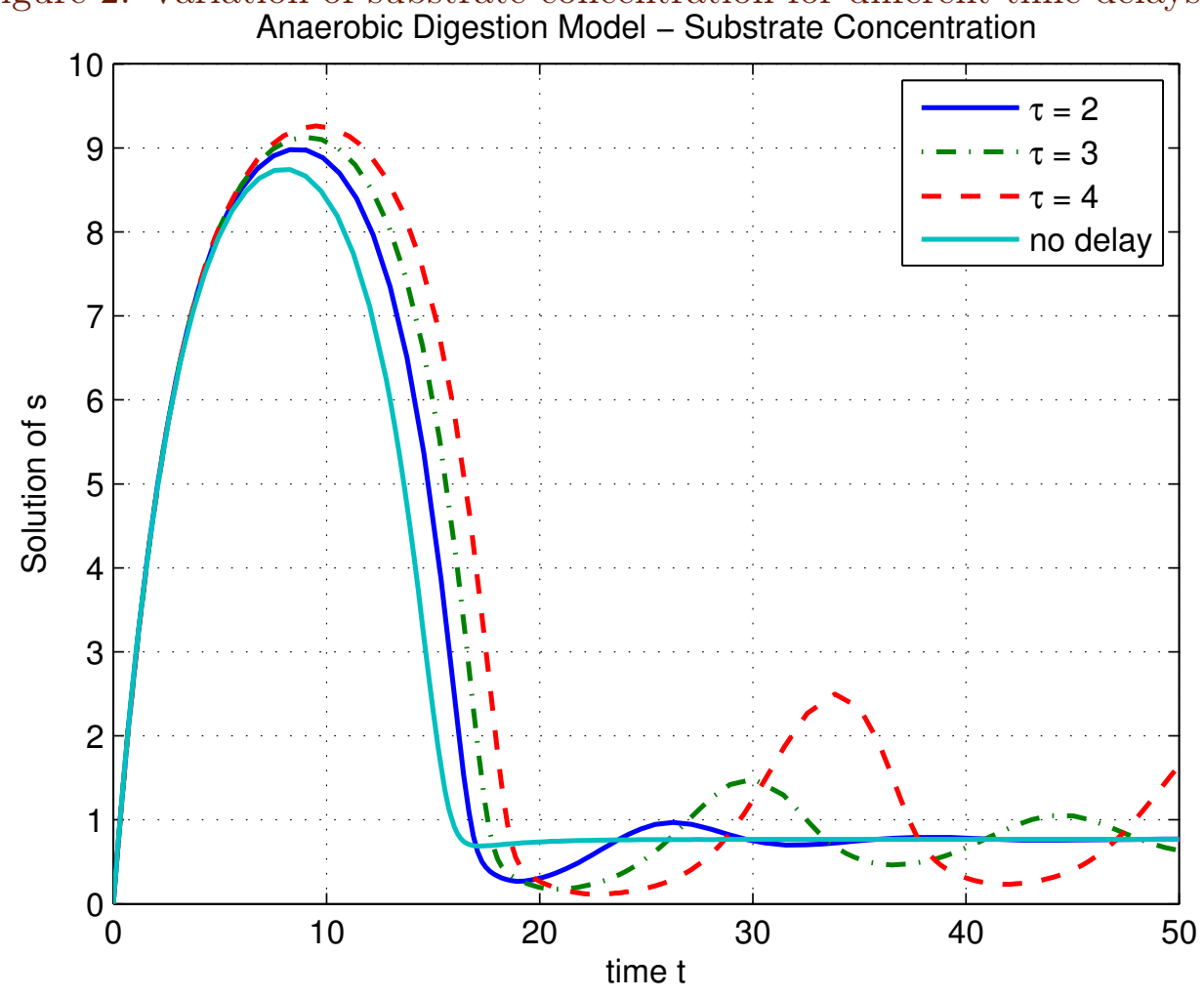

results for the substrate $s$. For no delay we see a high initial rise in substrate concentration caused by the influx of feed concentration. However, this decreases rapidly due to consumption of substrate by bacteria until a plateau is reached. This plateau corresponds to the steady state value of $s=0.76$ as shown in Table 1. Thus for non-delays, the profiles for $x$ and $s$ show a smooth transition to constant plateau values that are consistent with the critical values obtained in Section 2.1.3, as expected.

The other curves in Figures 1 and 2 show the $x$ and $s$ profiles obtained by the method described in Section 2.2 for three time delays of 2, 3 and 4. In all 
cases oscillations occur both in $x$ and $s$, with amplitudes increasing with $\tau$. In Figure 1, when there is a delay, we see an initial rise of biomass, which is consistent with the delay in the inhibition function $f$. However, we also notice a very steep rise in $x$ to a peak value that increases with $\tau$. This peak in $x$ corresponds to a decrease in $s$-almost to zero. This indicates that as $\tau$ increases there is a potential to stop the reaction; this halt occurs if $s$ is zero and the biomass $x$ dies. The oscillations do dampen out, and in time the profiles tend to the limiting values that occur in the non-time delay cases.

\subsection{Scenario two}

Here, we simulate a typical small scale laboratory reactor. Initially in this reactor we have a biomass but no substrate and the constant feed contains the substrate which provides a food source for the biomass. The rate of increase of biomass is given in equation (6) for the non-delay case, and in equation (20) for the time delay case. Evolution of the biomass is shown in Figure 3 where we see an initial decrease of $x$ as as the washout removes the biomass, but as time increases the kinetics growth term causes a consequent increase in $x$. Correspondingly, we see an sharp increase in $s$ with substrate in the feed; however, as the biomass increases this causes a decrease in $s$. This is consistent with equations (5) and (19).

In terms of time delay effects, we see no noticeable oscillations; however for $x$ in Figure 3 we do notice a delay in its increase as $\tau$ increases. Corresponding to this and as shown in Figure 4, after the initial rise, the $s$ value decreases slower with time, as $\tau$ increases.

In our study we endeavoured to introduce a cell type response with a delay in the kinetics inhibition term. We were guided by previous work, as discussed in Section 1. In our study of a typical anaerobic digester with data from Andrew [10], we showed that for a startup (Scenario one, Section 3.1) the reactor is stable. With the introduction of time delays, our analyses showed that oscillations occur which increase with increasing time delays. These 
Figure 3: Variation of biomass concentration for different time delays.

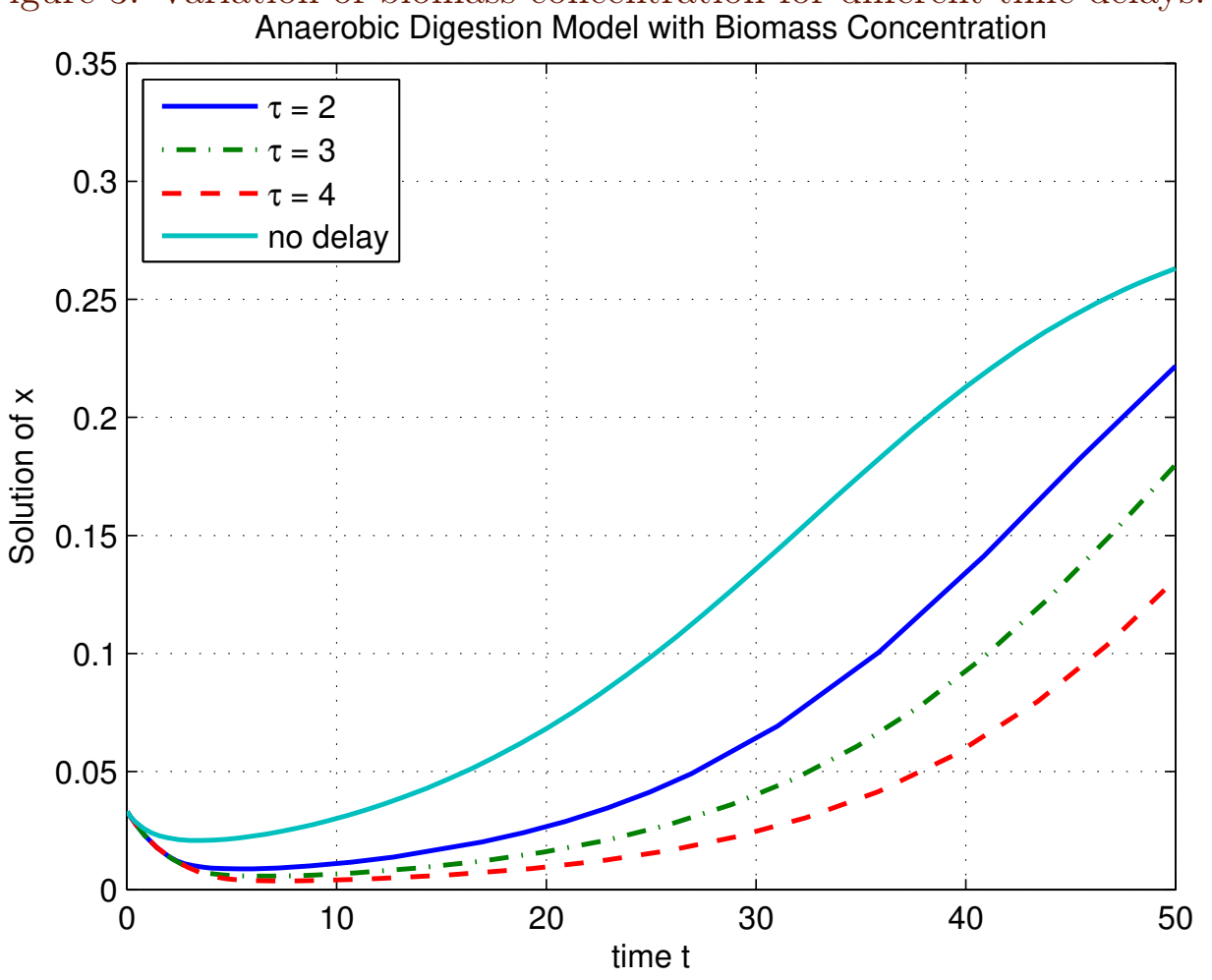

oscillation exacerbate the startup procedure by decreasing the substrate concentration to almost zero levels. In addition, we found for a small scale laboratory system (Scenario two, Section 3.2), that washout of the biomass is a possibility and the levels of increase in biomass through reaction is delayed with $\tau$, as expected. Such a dynamical study is important because it is difficult to measure the anaerobic response; and through appropriate modelling combined with appropriate experiments, a more accurate prediction can be achieved. 
Figure 4: Variation of substrate concentration for different time delays.

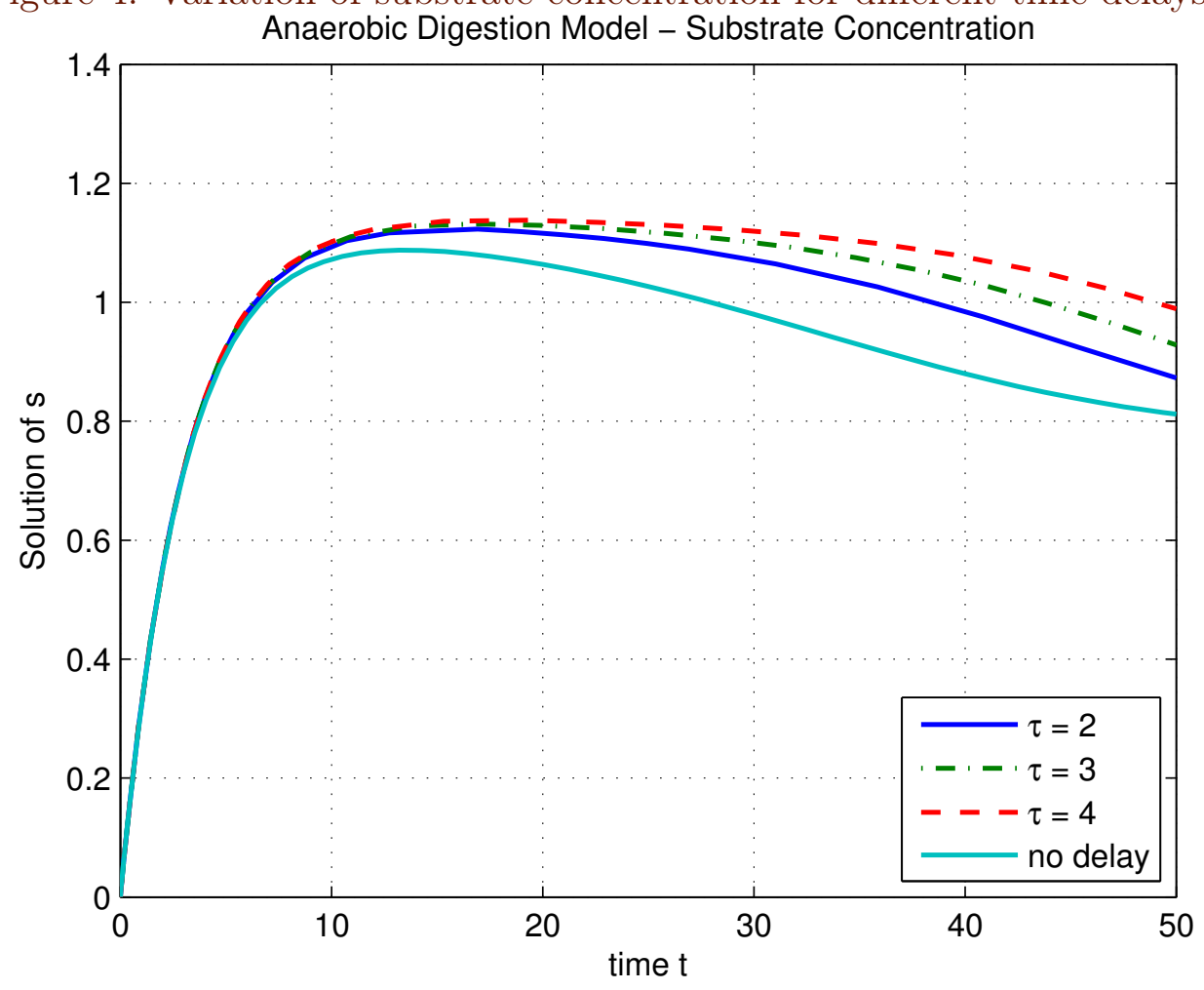

\section{References}

[1] O. Bernard, Z. Hadj-Sadok, D. Dochain, A. Genovesi, J. P. Steyer. Dynamical model development and parameter identification for an anaerobic wastewater treament process. Biotechnol. Bioeng. 75:424-438, 2001. doi:10.1002/bit.10036 C144

[2] T. F. Thingstad and T. I. Langeland. Dynamics of chemostat culture: The effect of a delay in cell response. J. Theor. Biol., 48:149-159, 1974. doi:10.1016/0022-5193(74)90186-6 C144 
[3] J. R. Beddington and R. M. May. Time delays are not nessarily destabilizing. Math. Biosci. 27:109-119, 1975. doi:10.1016/0025-5564(75)90028-0 C144

[4] K. Y. San and G. Stephanopoulos. The effect of growth rate delays in substrate-inhibited kinetics on the optimal profile of fed-batch reactors. J. Theor. Biol. 28:356-361, 1986. doi:10.1002/bit.260280308 C144

[5] G. Stephanopoulos and K. Y. San. Studies on-line bioreactor identification. I. Theory. Biotechnol. Bioeng. 26:1176-1188, 1984. doi:10.1002/bit.260261006 C144

[6] A. A. Khan, A. J. Stacey and J. J. Shepherd. Optimization of methane output for an anaerobic waste digester. ANZIAM J. 54:C523-C539, 2013. http://journal. austms.org.au/ojs/index.php/ANZIAMJ/ article/view/6322 C144

[7] J. F. Andrews. A mathematical model for the continuous culture of microorganisms utilizing inhibitory substrates. Biotechnol. Bioeng. 10:707-723, 1968. doi:10.1002/bit.260100602 C144

[8] D. J. Batstone, J. Keller, I. Angelidaki, S. V. Kalyuzzhni, S. G. Pavlostathis, A. Rozzi, W. T. M. Sanders, H. Siegrist, and V. A. Vavilin. Anaerobic digestion model No. 1 (ADM1). Water Sci. Technol. 45(10):65-73, 2002 http://wst. iwaponline. com/content/45/10/65 C145

[9] K. J. Beers. Numerical methods for chemical engineering: Applications in Matlab. Cambridge, New York, USA, 2007. http://www. cambridge. org/us/academic/subjects/engineering/chemical-engineering/ numerical-methods-chemical-engineering-applications-matlab C147

[10] J. F. Andrews. The development of a dynamic model and control strategies for the anaerobic digestion process. In Mathematical Models in Water Pollution Control, A. James (ed). Wiley, 1978. C145, C149, C155 
[11] A. W. Bush and A. E. Cook. The effect of time delay and growth rate inhibition in the bacterial treatment of wastewater J. Theor. Biol. 63:385-395, 1976. doi:10.1016/0022-5193(76)90041-2 C150

[12] L. F. Shampine, I. Gladwell and S. Thompson. Solving ODEs with Matlab. Cambridge, New York, USA, 2003.

http://www. cambridge.org/us/academic/subjects/mathematics/ numerical-analysis/solving-odes-matlab?format=PB C151

\section{Author addresses}

1. A. A. Khan, Mathematical and Geospatial Sciences,School of Science, RMIT University, Melbourne, Australia.

mailto:ash.khan@rmit.edu. au

2. J. J. Shepherd, Mathematical and Geospatial Sciences,School of Science, RMIT University, Melbourne, Australia.

mailto: jshep@rmit.edu. au

3. A. J. Stacey, Mathematical and Geospatial Sciences,School of Science, RMIT University, Melbourne, Australia. mailto:stacey@rmit.edu.au 